\title{
Psychiatric Training in Avon-Integrated or Separate Posts?
}

H. E. Barnes. Consultant in Child and Adolescent Psychiatry, Bristol Royal Hospital for Sick Children; H. Gethin Morgan, Professor of Mental Health. University of Bristol; M. G. Barker. Consultant Psychiatrist. Barrow Hospital. Bristol: I. E. BaBiker, Consultant Psychiatrist, Glenside Hospital, Bristol.

General professional training in psychiatry under the eye of the College has changed rapidly over the past twenty years providing benefit, as well as upheaval, for trainee and trainer alike. Along with the increased structure of training has come a change in trainees' career planning - as in all branches of medicine, perhaps $\rightarrow$ so that less sampling of different specialties and earlier commitment to a final career specialty is now taking place. This, together with the more formalized and structured training stipulations, has led to the development of so-called integrated training schemes in many centres. In Avon it has been felt that such a scheme would be neither practical nor in the trainees' best interests; but a persistent call by a proportion of trainees has fuelled continuing debate over several years.

In order to weigh up the issues more clearly, we present both the trainees' views. as represented by their responses to questionnaire, as well as figures collected by the trainers over the years concerning career developments and the implication they have for training needs.

\section{Survey and results}

All psychiatric trainees in the grades of senior house officer. registrar and senior registrar (including those in Mental Handicap and Child Psychiatry) in the Avon area were asked to complete a questionnaire. Questions asked directly whether the trainee would prefer an integrated training scheme with linked SHO and registrar posts, as opposed to the present unlinked system involving separate appointments, and looked for possible reasons. Other questions asked if the trainee had chosen Bristol especially for its present unlinked form of training, and why; or if not, whether he had had any hesitation in applying here. Further questions related to general personal data, placements in psychiatry so far, attitudes to psychiatry as a career, and satisfaction with the various aspects of training.

At the time of the questionnaire there was a total of 36 trainees and we were able to obtain full responses from 34 . Fourteen of these were senior registrars, 10 registrars, and 10 SHOs. Eighteen were male and 21 were married. Four senior registrars had previously trained elsewhere: their views have been included, except where inappropriate to do so, and otherwise do not affect the balance of findings.

Over two-thirds (23) would have preferred an integrated SHO/registrar training scheme. Although the respondents differed considerably in age and experience in psychiatry, this preference was of a similar order in all sub-groups of significant size; and in women and men alike ( 63 per cent of female trainees; 72 per cent of male). Twenty-one trainees expressing this preference gave as a reason for doing so that such a training scheme would provide greater certainty regarding future placements, and felt that this was important professionally and per- sonally: 15 of them also gave as a reason that it would provide a more integrated and coordinated training. In fact, 12 trainees stated that they had hesitated before applying to train in Avon. although in only 4 cases was this purely on training grounds. Three trainees had only applied because of personal ties locally.

On the other hand, 10 trainees did not favour an integrated training scheme and 4 of these would not have applied here if there had been one in operation. The present organization of training with the need to seek re-appointment to registrar grade especially attracted 5 trainees to apply to Avon training hospitals; reasons given were lack of commitment to psychiatry at the outset, and the flexibility which permitted choice of different placements at the various stages of their career.

Attitudes and career interests, were, however, reported as having changed during training. Thus, 6 trainees (4 males) on entering their first psychiatry post, had been seeking only a short experience before a non-psychiatric career; but 5 of them subsequently developed a firmer commitment to psychiatry. A further 12 trainees ( 8 males) had a firm intention initially to pursue a career in psychiatry; and this intention remains for all but one of them. The final 16 trainees ( 6 male) had been less sure, looking for a short experience which might possibly lead to a career in psychiatry; 14 of them later became more committed to such a carcer.

These figures, of course, do not take into account those who left psychiatry for general practice or other specialty after a period and who were not included in the questionnaire survey. Figures kept by the two main training hospitals, however, fill in this gap. One hospital has recorded trainees' career interests at the time of taking up SHO posts (though in a few cases this was not their first post in psychiatry) and also recorded their subsequent career moves. Since 1974 there have been 69 SHOs appointed and 27 of these ( 39 per cent) stated at the outset that they were specifically interested in general practice; 18 in fact did subsequently go into general practice while 9 remained in psychiatry. The other 42 SHOs stated at the outset that they were interested in psychiatry (though only a small proportion said they felt totally committed); 38 did subsequently pursue a career in psychiatry while the remaining 4 went into other disciplines. These figures do not include 20 further SHOs over this period who were part of a general practice rotation scheme and who came for six months each: in all but one case they did not pursue a career in psychiatry subsequently. This means that 31 per cent of SHOs did not remain in psychiatry (excluding those on general practice rotation schemes). but pursued other careers, chiefly general practice. Although this figure is actually lower than for earlier years (50 per cent of SHOs in 1969-1973 period), there is not a continuing trend and recent figures from the other main training centre in Avon are almost identicat- 6 
out of 19 SHOs ( 32 per cent) over the past three years have not remained in psychiatry.

In the case of those trainees who have now left psychiatry we cannot provide any measure of satisfaction with the training received. The majority of those presently in training, however, have experienced an increasing commitment to a career in psychiatry, during the period of their training, as already shown above. This must be, at least in part, a reflection of the training received. However, of those who trained here, 56 per cent were dissatisfied to some degree with the present organization of training on account of finding the uncertainty about future placements unsettling. The other main reason given for dissatisfaction felt by 30 per cent of those who trained here was that the training experienced lacked integration and co-ordination.

As the main reason both for dissatisfaction at present and for preferring integrated training concerns uncertainty over future placements and posts, and the effect of this on their training and domestic circumstances, we think it may be relevant that figures collected by one hospital do identify a recent trend towards more competition for registrar posts, with fewer of their own SHOs being appointed to fill these posts. Some of this trend results from a two-way 'exchange' of trainees between the two main training centres; but there are in addition some appointments of trainees from outside the area to registrar posts.

\section{Discussion}

On the face of it then an ideal scheme would provide an integrated training with linked $\mathrm{SHO} /$ registrar posts for those trainees who wish to pursue a career in psychiatry, while also providing short experience SHO posts for those who did not (in addition to any GP rotation scheme that is operating). However, in practice there are many arguments against such an arrangement and these were raised by trainees themselves as well as their trainers.

Chiefly there is concern to avoid a two-tier structure with the 'best placements' and the most attention being given to the 'career' trainees, leaving the other trainees in short experience posts feeling somewhat 'second best'. The only effective way to be sure of avoiding this would be to have no differentiation at all from the outset; but in that case sufficient registrar placements would need to be available to guarantee all SHOs continued training if required. Where this is not presently the case, as in Avon, the required change cannot realistically be expected in the current economic climate.

This leaves a choice between, on the one hand an integrated training for future psychiatrists which might cater poorly for others, and on the other hand an unlinked training structure with necessary reapplication for registrar posts. The advantages and disadvantages of each are now summarized.

An integrated training scheme might theoretically offer a great variety of training experience through advanced planning and co-ordination of a given trainee's programme for the entirety of his training. In practice this advantage would be limited by the complexities of co-ordinating the programmes of all the trainees; and further limitations would occur if there was to be any flexibility to determine placements in the light of developing experience and interests as the training proceeded. The major advantage of this type of training. which has already been picked out. is the provision of greater security for trainees so as to better plan their personal and social lives. It is possible too that the personal aspects of their training might benefit from time to settle in psychiatry without the pressure of exams and the necessity of presenting their case for a registrar appointment, and so allowing better for the development of research and other special interests.

The disadvantage of an integrated training scheme with linked posts appears to be the failure to allow for changing training needs and career interests. There is an encouragement to earlier commitment to specialization and discouragement of those uncertain about their careers but who might wish to sample work in psychiatry without a necessary career commitment to it.

An unlinked scheme with distinct SHO and registrar posts permits effective differentiation of preliminary general psychiatry SHO type of experience from the more specialized registrar placements. Flexibility is an advantage of this system allowing training to be tailored to individual needs.

The disadvantage of the 'separate post' training is that it cannot provide as much security for trainees; but there are ways in which SHO and registrar posts can be dovetailed to keep personal and domestic disruption at a minimum. For example, given that appointment to registrar posts in centres operating an integrated scheme are of necessity an internal matter, there is then no reason why such appointments should not be offered preferentially to local SHOs in the other areas also.

\section{Conclusion}

Overall we remain impressed by the advantages of a training scheme with distinct SHO and registrar posts and by the disadvantages of integrated training schemes. We acknowledge. however, the important issue that the development of integrated training schemes has raised of the advantage to trainees of minimizing personal and domestic disruption at a sensitive period of their training. Training centres which maintain a 'separate post' structure must clearly give a great deal of thought to this matter and institute at the earliest possible time that is economically viable changes which will help those trainees interested in a psychiatric career to have a training which is both settled and well planned. With such development 'separate post' schemes might approach the ideal as near as is presently possible. More doctors would find it attractive to have some psychiatric training without commitment; and not only do our figures show what a valuable pool for recruitment into psychiatry this group provides, but moreover potential general practitioners should have the opportunity of first class SHO posts in psychiatry. Attention to individual needs in training, along with flexibility, should be provided for all trainees, while those deciding to continue with full training in psychiatry at registrar level would hopefully experience the minimum disruption when applying for such posts. 